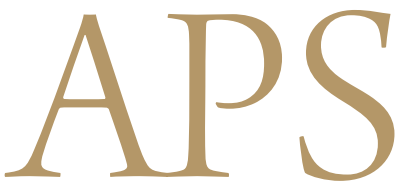

Archives of Plastic Surgery

\title{
Radiation Therapy Following Total Keloidectomy: A Retrospective Study over 11 Years
}

\author{
Kyuhee Kim ${ }^{1}$, Daegu Son ${ }^{1}$, Jinhee Kim ${ }^{2}$ \\ Departments of ${ }^{1}$ Plastic and Reconstructive Surgery and ${ }^{2}$ Radiation Oncology, Keimyung University Dongsan Medical Center, Keimyung \\ University School of Medicine, Daegu, Korea
}

Background Radiotherapy treatment after keloidectomy is known to be an effective method for reducing the rate of recurrence. However, to date, the appropriate total radiation dose and fractionation have not yet been confirmed. The authors performed a retrospective analysis to identify the appropriate radiation dose and fractionation in post-keloidectomy radiotherapy. Methods From May 2000 to February 2011, postoperative radiotherapy was performed on 39 lesions in 28 patients after total keloidectomy. The keloid lesions were confined to the ear lobes. Between May 2000 and May 2004, 14 keloids were treated with surgical excision, followed by a total radiation dose of 1,200 cGy in three fractions over four to five days (group 1). Between June 2004 to February 2011, 25 keloids were treated with surgical excision, followed by a total radiation dose of 1,500 cGy in three fractions over four to five days (group 2). Patients were given a survey asking them to report their experiences regarding reoperation, recurrence of symptoms, recurrence of the lesion, and satisfaction with the operation.

Results Of the 28 patients who were treated, 20 underwent follow-up. Group 2 had more cases showing elevation with erythematous changes, whereas group 1 had more cases showing progressive stages of elevation than group 2 . These differences were statistically significant. Moreover, a correlation was observed between the level of keloid elevation and the extent of symptoms.

Conclusions We suggest 1,500 cGy of radiation in three fractions following keloidectomy for ear lobe keloids. A further randomized study is needed to assess the recurrence of keloids after radiotherapy.

\section{Keywords Keloid / Radiation / Ear}

\author{
Correspondence: Daegu Son \\ Department of Plastic and \\ Reconstructive Surgery, Keimyung \\ University School of Medicine, \\ 56 Dalseong-ro, Jung-gu, \\ Daegu 41931, Korea \\ Tel: +82-53-250-7636 \\ Fax: +82-53-255-0632 \\ E-mail: handson@dsmc.or.kr
}

This article was presented at the 71st Congress of the Korean Society of Plastic and Reconstructive Surgeons on November 1-3, 2013 in Seoul, Korea.

No potential conflict of interest relevant to this article was reported.

Received: 23 Apr 2015 • Revised: 5 Jun $2015 \bullet$ Accepted: 19 Jun 2015

pISSN: 2234-6163 • elSSN: 2234-6171 • http://dx.doi.org/10.5999/aps.2015.42.5.588• Arch Plast Surg 2015;42:588-595

\section{INTRODUCTION}

Although keloids can occur anywhere on the body, keloids in the facial area are conspicuous. The cosmetic effects of keloids can result in decreased self-esteem among patients and a significant decline in their quality of life. The treatment of keloids by surgical excision has been reported to result in recurrence rates from $45 \%$ to $100 \%$ [1], which has led to the development of various adjuvant therapies in order to reduce the recurrence rate. Earlobe keloids have drawn much more attention than keloids occurring in other areas, because the incidence of keloids after ear piercing is $2.5 \%$ [2].

Surgical excision, postoperative radiotherapy, steroid injection, sheeting, and pressure therapy have been widely used to treat 
keloids. In particular, radiation therapy has become an important adjuvant therapy for keloid scars, and keloid scars are currently the most common type of benign disease treated by radiotherapy [3].

In our previous study, we found that postoperative radiotherapy using the plane surveying method after keloidectomy results in a size reduction of $55 \%$, and the effectiveness of the treatment is enhanced when it is performed within 24 hours of surgery $[4,5]$. In many situations, radiation is initiated immediately after surgery, and the total dose is limited to 20 Gy over several administrations [6,7]. A total dose of $15 \mathrm{~Gy}$ has been used most frequently, according a survey by Niessen et al. [8].

People with darkly pigmented skin are 15-fold more likely to develop keloids than those with white skin [8]. In contrast to keloids in the chest wall, where the conditions of high tension affect the recurrence rate, earlobe keloids have a significantly lower recurrence rate after resection than those occurring at other sites, both with and without preoperative therapy [6]. Therefore, the total radiation dose should be optimized based on the racial background of the patient and the anatomical area of the keloid lesion. Due to the lack of guidelines for the optimal dose of radiation, a long-term follow-up study is required to determine the optimal total radiation dose.

Although Ogawa et al. [9] have evaluated the effective total dose of radiation necessary for reducing the recurrence rate after earlobe keloidectomy, their study has several limitations. First, the objective criteria for recurrence were not clear, as recurrence was assessed depending on the doctor's subjective judgment. Second, only the re-elevation of the keloid mass after post-keloidectomy radiotherapy was investigated, with no analysis of the recurrence of symptoms such as pruritus or pain.

In this study, we investigated not only the elevation of keloids but also the degree of re-elevation of the keloid and the recurrence of symptoms. The optimal dose of radiation was assessed by reviewing patient records and administering a survey asking patients to assess the elevation of keloids and the recurrence of symptoms after the surgical excision of keloid lesions followed by irradiation with a dose of $1,200 \mathrm{cGy}$ or $1,500 \mathrm{cGy}$.

\section{METHODS}

\section{Subjects}

After receiving Institutional Review Board approval (IRB No. 2015-03-021-001), this retrospective study was conducted via a patient telephone survey and chart review. All patients who had received radiotherapy after the surgical excision of keloids between May 2000 and February 2011 were identified. The exclusion criteria were as follows: female patients who were likely to become pregnant, patients who had previously received radiation therapy in the head and neck area, and patients who underwent less than one year of follow-up.

Between May 2000 and May 2004, 13 keloids were treated with surgical excision, followed by a total radiation dose of 1,200 cGy in three fractions over four to five days (group 1). Between June 2004 to February 2011, 25 keloids were treated with surgical excision, followed by a total radiation dose of $1,500 \mathrm{cGy}$ in three fractions over four to five days (group 2).

One patient in group 1 underwent reoperation. In 2006, a new protocol was implemented, increasing the total dose to 1,500 cGy in three fractions. In order to determine the optimal dose of postoperative radiotherapy, we conducted a statistical comparison of the results of the 1,200 cGy protocol before May 2004 with those of the 1,500 cGy protocol after June 2004.

Thereafter, for long-term follow-up, patients were surveyed via telephone and asked to report their experiences regarding reoperation, recurrence, re-elevation of the keloids, and their satisfaction level.

\section{Surgical technique}

Under local anesthesia, 1:100,000 epinephrine mixed with 1\% lidocaine was infiltrated around the keloid. The keloidectomy involved the excision of all keloid tissue and an area of surrounding normal tissue ( 0.5 to $1.5 \mathrm{~mm}$ ) using a surgical knife with a No. 15 blade. A simple buried suture was placed in the subcutaneous tissue using an absorbable filament, and a simple interrupted skin suture using a non-absorbable filament was put in place without tension. After surgical resection of the mass, we applied a semi-occlusive compressive wound dressing using a foam dressing (Mepilex Lite, Molnlycke Health Care, Gothenburg, Sweden) and a cotton ball to prevent hematoma around the surgical wound, and on the day after surgery, adjuvant postoperative radiotherapy was initiated within 24 hours of the procedure. We used a moderate-energy electron beam ( 6 to $9 \mathrm{MeV}$ ) and an overlying bolus in the radiotherapy treatment. Radiation was delivered by a linear accelerator (Siemens Co., Munich, Germany) with a bolus. Underlying shielding ( $3 \mathrm{~mm}$ of lead) was used to protect the normal tissue during treatment of the full thickness of the earlobe. The keloids were classified into two groups. Group 1 was treated with a lower total radiation dose of $1,200 \mathrm{cGy}$, delivered in three fractions over three days starting on the day after surgery. Group 2 was treated with a higher total radiation dose of $1,500 \mathrm{cGy}$, delivered in three fractions over three days starting on the day after surgery. The sutures were removed during the second postoperative week. 


\section{Evaluation}

In each group, the results of treatment were assessed using a chart review. Patients were examined at one day, one week, one month, and six months after surgery. Information was recorded about patients who showed signs of recurrence involving clinical evidence of a mass, such as recurrence of symptoms or recurrence of mass elevation). Side effects and complications such as telan- giectasia or skin pigmentation were also noted. Patient demographics (gender, age), the amount of postoperative electronbeam irradiation administered, and complications were recorded.

In the telephone survey that was used to perform long-term follow-up, the patients were asked about the following four topics: history of reoperation and interval between the original operation and reoperation, recurrence of symptoms, recurrence of

\section{Fig. 1. Questionnaire for patients after surgery}

Via a telephone survey, the following four issues were assessed: history of reoperation and time to reoperation, recurrence of symptoms, recurrence of the lesion, and satisfaction with the operation.

Questionnaire for patients after surgery

- Name (initials)

- Date

Please answer the following questions about recurrence and satisfaction after ke loid surgery

\section{Reoperation}

1.1 Have you had a reoperation in this hospital or another hospital after surgery? $\square$ Yes $\square$ No 1.2 If you had a reoperation in another hospital, when did you have it?

( ) years and ( ) months after surgery

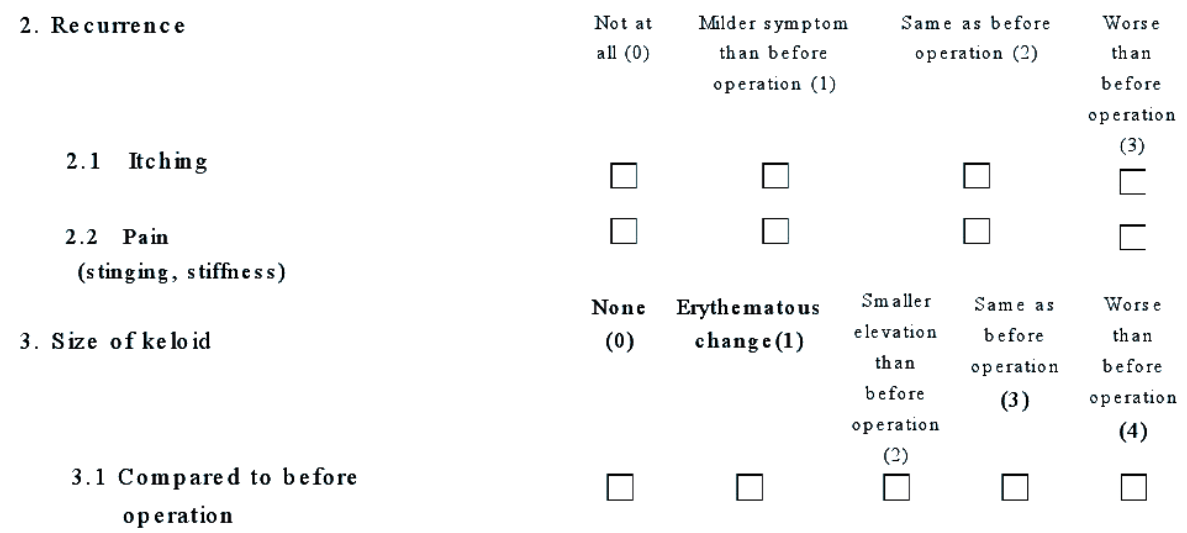

4. Satisfaction with operation and radio the rapy

4.1 Are you satis fied with the combination of surgery and radiotherapy?

$$
\text { Very Unsatisfied (2) Neutral Satisfied (4) Very }
$$$$
\text { unsatis fie d (1) (3) } \quad s \text { atis fied (5) }
$$

\begin{tabular}{lllll}
$\square$ & $\square$ & $\square$ & $\square$ \\
\hline
\end{tabular}

Patient name:

Date :

Le gal repres entative

Relation:

(If necessary)

Signature

Date:

Princ ip al investig ator/Co - inve stig ator

name :

Signature :

Date : 
the lesion, and level of satisfaction with the operation. The recurrence of symptoms, recurrence of the lesion, and time between surgery and onset of symptoms were quantitatively analyzed. The following scoring system was adopted to assess the severity of symptoms: no symptoms (0), symptoms milder than before treatment (1), symptoms equivalent to those experienced before treatment (2), and symptoms worse than those observed before treatment (3). The elevation of the keloid was scored as follows: no elevation (0), erythematous changes only (1), elevation lower than that observed before treatment (2), elevation similar to that observed before treatment (3), and elevation higher than that observed before treatment (4).

\section{Statistical analysis}

The two-sample t-test and the chi-squared test were used to analyze the differences between the two groups in SPSS ver. 22.0 (IBM Co., Armonk, NY, USA). P-values $<0.05$ were considered to indicate statistical significance. Data were reported as medians and ranges.

\section{RESULTS}

\section{Subjects}

A total of 38 lesions in 27 patients who underwent postoperative radiotherapy after total keloidectomy between May 2000 and February 2011 were identified. Between May 2000 and May 2004, 13 lesions were treated with surgical excision, followed by a total radiation dose of $1,200 \mathrm{cGy}$ in three fractions over four to five days (group 1). Between June 2004 and February 2011, 25 keloids were treated with surgical excision followed by a total radiation dose of $1,500 \mathrm{cGy}$ in three fractions over four to five days (group 2). Of the 27 patients with 38 lesions, 19 patients with 22 lesions responded to the questionnaire, yielding a response rate of $71.4 \%$ (Fig. 1). Responses were provided corresponding to six of the 13 keloids included in group 1 and 16 of the 25 keloids included in group 2. The characteristics of the patients who responded are summarized in Table 1 . The mean age of all respondents was 57.68 years (range, 15 to 72 years). The median age was 31 years (range, 25 to 41 years) in group 1 , and 26.68 years (range, 15 to 72 years) in group 2 . The minimum followup period was 15 months. The median follow-up period in group 1 was 132 months (range, 132 to 160 months), and the medium follow-up period in group 2 was 49.25 months (range, 15 to 124

Table 1. Demographic information of patients with earlobe keloids treated with radiation therapy following total keloidectomy: group 1 and group 2

\begin{tabular}{|lccc|}
\hline Variable & $\begin{array}{c}\text { Group 1 } \\
(\mathbf{1 , 2 0 0} \text { cGy) }\end{array}$ & $\begin{array}{c}\text { Group 2 } \\
(\mathbf{1 , 5 0 0} \text { cGy })\end{array}$ & P-value \\
\hline No. of keloids & 6 & 16 & - \\
Mean patient age (yr) & $31.67 \pm 3.1$ & $26.69 \pm 3.51$ & 0.064 \\
Left ear/right ear & $4 / 2$ & $9 / 7$ & 0.658 \\
Mean follow-up period (mo) & $132 \pm 14.53$ & $49.25 \pm 27.01$ & 0.001 \\
Mean diameter of keloid (cm) & $2.33 \pm 0.81$ & $1.59 \pm 0.92$ & 0.070 \\
Total radiation dose (cGy) & 1,200 & 1,500 & - \\
Fraction (time) & 3 & 3 & - \\
\hline Values are presented as number or mean \pm standard deviation. & \\
\hline
\end{tabular}

\section{Table 2. Results of our study}

\begin{tabular}{|lcc|}
\hline Variable & Group 1 (\%) & Group 2 (\%) \\
\hline No. of patients & 6 & 16 \\
Keloid reoperation rate & $1(16.7)$ & 0 \\
Recurrence of itching sensations & $5(83.3)$ & $11(68.7)$ \\
$\quad$ Not at all & $1(16.7)$ & $5(31.3)$ \\
Milder than before operation & 0 & 0 \\
Same as before operation & 0 & 0 \\
Worse than before operation & $5(83.3)$ & $12(75.0)$ \\
Recurrence of pain & $1(16.7)$ & $4(25.0)$ \\
$\quad$ Not at all & 0 & 0 \\
Milder than before operation & 0 & 0 \\
Same as before operation & & 0.494 \\
Worse than before operation & $3(50.0)$ & $8(50.0)$ \\
Size of keloid & 0 & $7(43.8)$ \\
None & $3(50.0)$ & $1(6.3)$ \\
Erythematous change & 0 & 0 \\
Smaller elevation than before operation & 0 & 0 \\
Same as before operation & $4.33 \pm 0.81$ & 0.678 \\
Worse than before operation & $33.00 \pm 16.09$ & $19.06 \pm 1.12$ \\
Satisfaction level of patients (1: very unsatisfied, 5: very satisfied) & & 0.027 \\
Mean recurrence interval (mo) & & 11.06 \\
\hline P-values for continuous variables were obtained by using the Wilcoxon signed rank test. P-values for categorical variables were obtained by using the chi-squared test. \\
\hline
\end{tabular}


months), resulting in an overall mean follow-up period of 79.1 months. The average diameter of the lesions was $2.33 \mathrm{~cm}$ in group 1 and $1.59 \mathrm{~cm}$ in group 2.

\section{Keloid reoperation rates}

One patient in group 1 underwent reoperation one year and eight months after the first surgery, due to the development of keloids equal in size to the pretreatment keloids along with discomfort caused by the recurrence of symptoms such as pain and itching. No patients in group 2 underwent reoperation (Table 2).

\section{Recurrence of symptoms}

Itching sensation

In group 1, five patients (83.3\%) reported no itching sensation, and one patient (16.7\%) exhibited symptoms that were milder than those experienced before treatment. In group 2, eleven patients $(68.7 \%)$ reported no itching sensation, and five patients (31.3\%) reported milder symptoms than they experienced before treatment. No patients in either group reported experiencing symptoms worse than those experienced before treatment.

\section{Pain}

In group 1, five patients (83.3\%) experienced no pain, and one patient (16.7\%) experienced pain to a lesser extent than before treatment. In group 2, twelve patients (75\%) experienced no pain, and four patients (25\%) experienced milder pain than be- fore treatment. Similar to the results regarding itching, none of the patients reported symptoms worse than those experienced before treatment.

\section{Re-elevation of keloids}

In group 1, no elevation was observed in three cases (50\%), and mild elevation, to a lesser extent than was observed before treatment, occurred in three cases (50\%). In group 2, no elevation was observed in eight cases (50\%) (Fig. 2), and mild erythematous changes were observed in seven cases (43.8\%). In one case (6.3\%), elevation less than that which occurred before treatment was noted (Fig. 3). In group 2, many patients showed erythematous changes, whereas in group 1, more cases showed progressive stages of elevation. These differences were statistically significant $(\mathrm{P}=0.027)$.

\section{Mean recurrence interval}

In group 1, symptoms recurred between 18 and 50 months (mean, 33 months) after surgery. In group 2, symptoms recurred between 10 and 34 months (mean, 19.43 months) after surgery.

\section{Satisfaction level of patients}

Patients' satisfaction levels were assessed using a visual analog scale of 1 to 5 , with 5 indicating the highest level of satisfaction. Group 1 reported a satisfaction level of 4.33 points, and group 2 reported a satisfaction level of 4.06 points.

\section{Fig. 2. A case of keloid with radiotherapy}

(A) Preoperative view. (B) Immediate postoperative image. She underwent keloidectomy under local anesthesia. Immediate radiotherapy with a dose of 1,500 cGy in three fractions was initiated on the day after the operation. (C) Three years postoperatively, no recurrence of the elevation of the mass was noted.
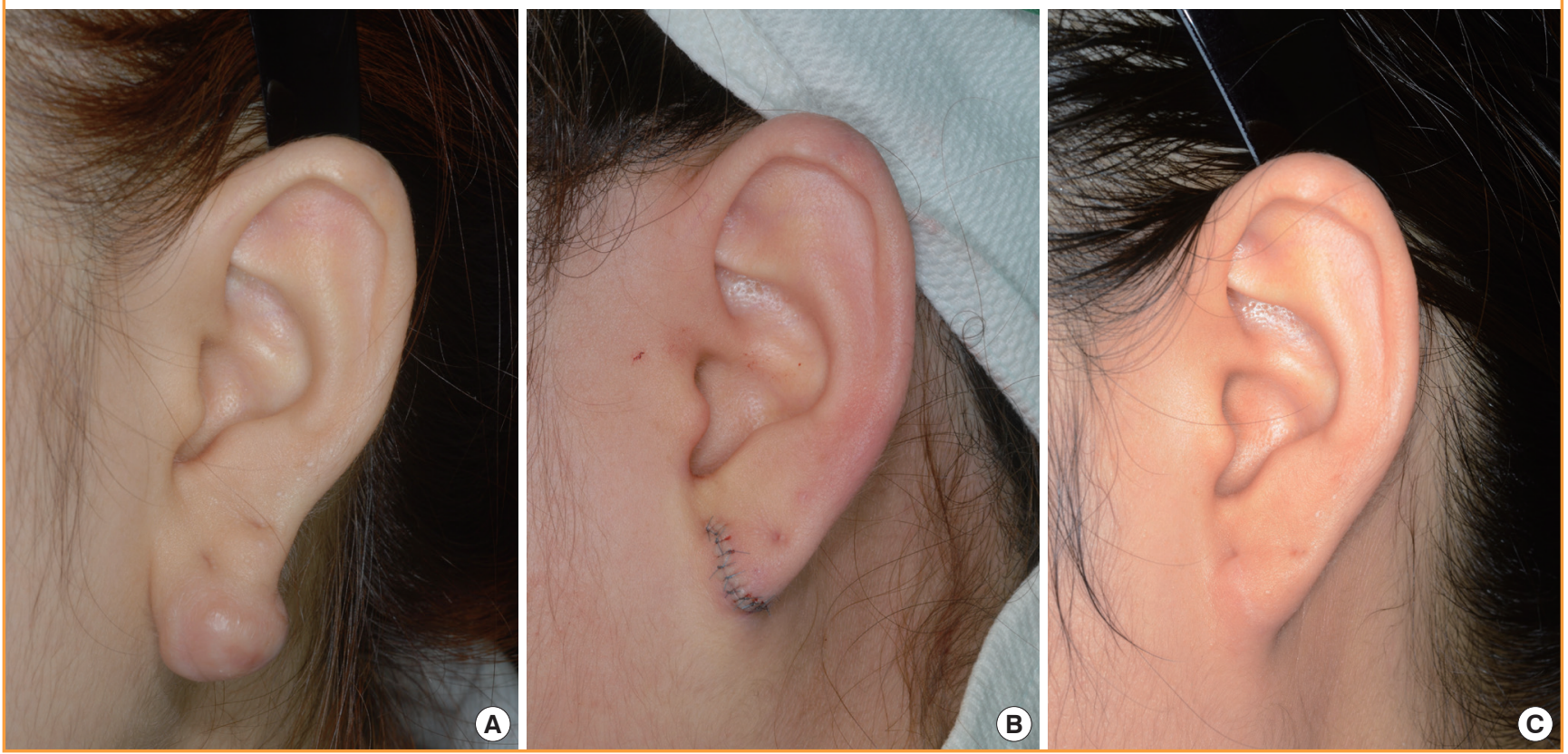


\section{Fig. 3. A case of keloidectomy with radiotherapy}

(A) Preoperative view. (B) Immediate postoperative image. She underwent keloidectomy under local anesthesia. Immediate radiotherapy with a dose of 1,500 cGy in three fractions was initiated on the day after the operation. (C) Three months postoperatively, no recurrence of elevation of the mass was noted. (D) 24 months postoperatively, mild elevation of the mass on her earlobe was noted. She reported an itching sensation at one point on her ear.
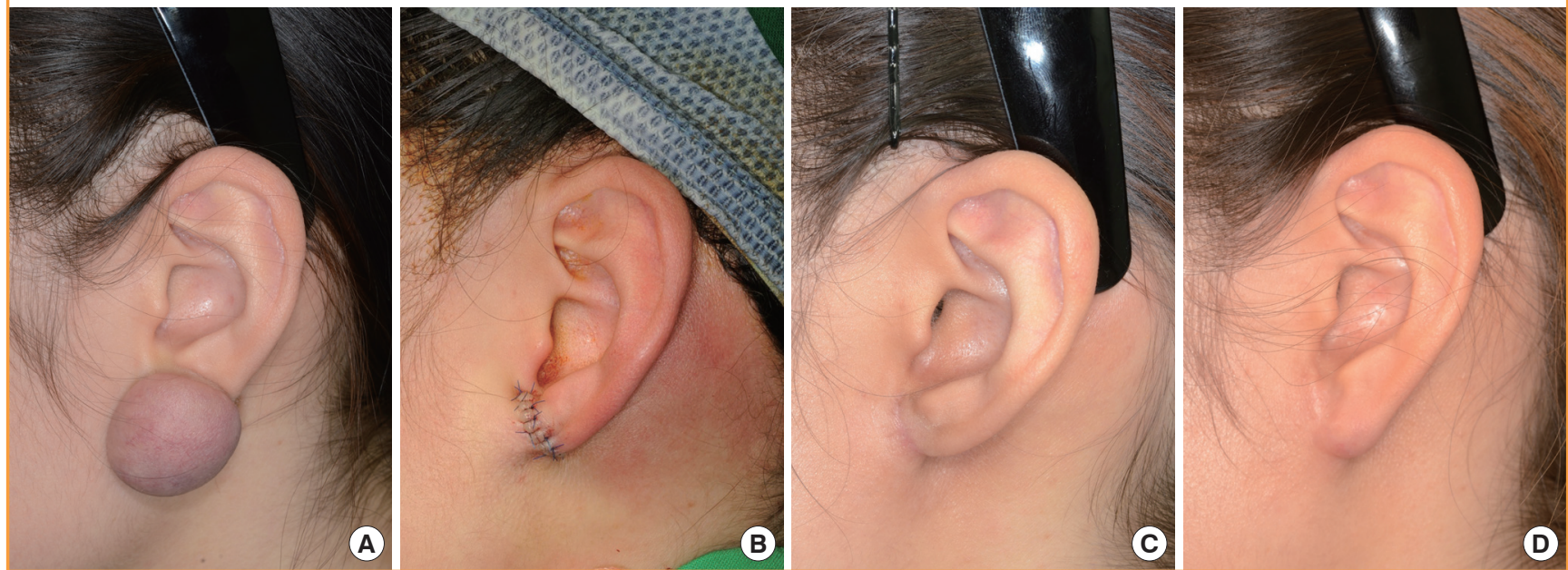

\section{Postoperative adjuvant therapy and management}

Patients who exhibited erythematous changes or mild elevation after the operation were provided with the option of adjuvant therapy with triamcinolone injections or magnetic pressure therapy, subject to their consent. In group 1, among the four cases in which mild elevation occurred, we injected triamcinolone into one lesion and used magnetic pressure therapy in one other case. In group 2, among the seven cases in which erythematous changes occurred, we injected triamcinolone in one case and performed magnetic pressure therapy in two other cases. We applied magnetic pressure therapy in one keloid in which mild elevation occurred.

\section{Complications}

None of the patients in either group reported hyperpigmentation or hypopigmentation, delayed wound healing, chondritis, or ulceration. None of the patients reported being diagnosed with cancer.

\section{DISCUSSION}

Postoperative radiotherapy was administered using a 6 to $9 \mathrm{MeV}$ electron beam to deliver a total dose of $1,500 \mathrm{cGy}$ or 1,200 cGy in three fractions for three days, starting on the first postoperative day, with full shielding to protect the normal tissue. Some authors have suggested single fractions of up to 10 to $15 \mathrm{~Gy}$, and have reported excellent results and cosmesis $[10,11]$. Despite the very low risk of late tissue complications such as atrophy, telangiectasia, hypopigmentation, and subcutaneous fibrosis com- plications, the incidence rate of these late tissue complications can increase with the dose per fraction [12]. Therefore, we employed a dose of 400 to $500 \mathrm{cGy}$ per fraction.

Kovalic and Perez [13] reported a control rate of $73 \%$ over an average of 12.8 months of follow-up after a total dose of 1,200 cGy was delivered in three fractions over three days after surgery. Levy et al. [14] reported that a total dose of 1,800 cGy delivered in five to six fractions over 12-14 days, starting within 24 to 48 hours of the operation, resulted in a cure rate of $88 \%$ over a follow-up period ranging from six to 20 months.

In a study by Ogawa et al. [6] in which keloidectomy was only performed in earlobe keloids defined as lesions according to the authors' keloid protocol, they recommended postsurgical radiotherapy with a dose of $1,000 \mathrm{cGy}$ in two fractions, based on their finding that no statistically significant difference was observable in recurrence rates after postsurgical radiotherapy depending on the use of a total dose of $1,000 \mathrm{cGy}$ in two fractions or 1,500 cGy in three fractions.

Although recurrence rates were discussed in the studies presented above, evaluating the results of therapy by performing a simple comparison of recurrence rates is not sufficient, because each study applied its own criteria to define the recurrence rate. For this reason, we compared the re-elevation of keloids by scoring the level of keloid elevation. Moreover, in contrast to Ogawa's report, surveillance results analyzing the recurrence of symptoms were included in this study. Our study showed that group 2 , which received a higher total radiation dose, had more cases showing elevation with erythematous changes, whereas group 1 had more cases showing progressive stages of elevation $(\mathrm{P}=0.027)$. 
The correlation between the level of keloid re-elevation and the recurrence symptoms of pain or itching was statistically significant $(\mathrm{P}=0.020, \mathrm{P}=0.036)$. No patient experienced a recurrence of symptoms without accompanying re-elevation of the keloid. Further prospective studies are needed to investigate whether pain and itching symptoms precede the elevation of a keloid.

Postoperative electron-beam irradiation may control collagen synthesis by inhibiting the promotion of abnormally activated and normal fibroblasts [15]. Kal et al. [16] also demonstrated a dose-effect relationship between radiation dose and keloid recurrence by analyzing the reported recurrence rates of keloids and pterygia after postoperative radiation therapy. Determining the optimal dose of radiation is critical because radiotherapy is potentially carcinogenic, although higher doses of radiation can reduce the rate of recurrence. Malignant tumors that develop on the earlobe have not yet been reported. A case of carcinoma of the thyroid after irradiation with a dose of 12 Gy to the chin has been reported [17], and a case of breast cancer after superficial X-ray treatment with a dose of $13 \mathrm{~Gy}$ to the breast has been reported [3]. The crude risk from the published data that we have evaluated is five reported cancers out of 6,741 reported treatments $(0.074 \%)$ [10]. Only one case, involving fibrosarcoma, may have resulted from a malignant change in the keloid; the other four cases showed carcinogenesis in the surrounding normal tissues, but a causal relationship between the treatment and carcinoma was not established [18]. We did not encounter any such cases among our patients, who were followed for up to 160 months. Since most patients who receive radiotherapy for keloid treatment are young patients who are intensely interested in their cosmetic appearance, they should be fully informed that even low-dose adjuvant radiotherapy has the potential to cause radiation-induced malignancy, and therefore, healthcare providers should protect the surrounding tissues with full shielding. Since choosing the optimal radiation dose without affecting the recurrence rate is beneficial to the patients, the current study addressed this critical point. In this study, radiation doses of 1,200 cGy and 1,500 cGy were compared. Although no statistical significance was observed in the recurrence of symptoms, worse outcomes were observed in the 1,500 cGy group than in the $1,200 \mathrm{cGy}$ group regarding the mean recurrence interval and the satisfaction levels of the patients. However, we found that the 1,500 cGy group experienced a significantly lower extent of reelevation of the keloids. Re-elevated keloids on the ear lobe that show a lesser degree of elevation than before the operation can be managed with adjuvant therapy such as magnetic ring compression or steroid injection. Therefore, we focused on the extent of re-elevation of the keloid, not on the frequency of recur- rence. Although the differences in the recurrence of pain or itching symptoms between the two groups were not statistically significant, we recommend irradiation with a dose of 1,500 cGy after total keloidectomy, especially for earlobe keloids, considering that there was a correlation between the level of keloid elevation and symptoms such as itching or pain.

This study had some limitations. First, the number of patients who included in this study is too small only 19 patients responded. Second, since this was a retrospective study, the mean follow-up period in group 1 (before 2004) was longer than that in group 2 (after 2004). Nevertheless, group 2 had an adequate follow-up period, because the mean follow-up period in group 2 was 49 months and the mean interval between the operation and recurrence was 24 months. Third, the results have limited objectivity because the dataset regarding long-term follow-up was collected via telephone surveillance, and the patients subjectively evaluated the level of keloid elevation. However, longterm follow-up with telephone surveillance was essential because it is difficult to follow outpatients for a period longer than one year, the mean interval of recurrence was 25 months, and most patients who experienced recurrence did not visit the clinic. The mean interval of recurrence was 37 months in group 1 and 19 months in group 2, supporting the need for a follow-up period of at least two or three years, which is in agreement with the findings of a previous study [12]. Finally, a prospective study with a wider range of radiotherapy techniques is necessary in order to determine the most effective radiotherapy dosage. Variations in the amount of radiation exposure in the same radiation dosage should also be studied. This study was a retrospective study performed in advance of a future prospective study, and the purpose of this study was to assess the effectiveness of radiotherapy following total keloidectomy by comparing radiation doses of $1,200 \mathrm{cGy}$ and 1,500 cGy. The authors studied 11 years of retrospective data on two types of radiation therapy that have been used globally. We assessed not only the degree of keloid re-elevation but also the recurrence of symptoms. We concluded that although re-elevation of the keloids occurred more frequently in the 1,500 cGy group than in the 1,200 cGy group, the degree of re-elevation of the keloids was significantly lower in the 1,500 cGy radiation group. Moreover, a correlation was observed between the extent of keloid elevation and symptoms such as itching or pain. We found that radiotherapy following keloidectomy is a safe treatment modality due to the absence of complications such as the occurrence of a malignant neoplasm. Based on our study, a further prospective study should be carried out, studying a diverse range of radiotherapies and amounts of radiation exposure, including a higher radiotherapy dosage.

As also indicated by previously published data, surgical extral- 
esional excision followed by radiotherapy is still a good option for preventing the recurrence of keloids. There are no late softtissue or epidermal side effects of radiotherapy. Considering the alleviation of symptoms such as keloid re-elevation, pain, and itching after surgery, we recommend surgical excision and postsurgical radiation therapy with a dose of $1,500 \mathrm{cGy}$ in three fractions. The safety and effectiveness of postoperative radiation therapy after keloidectomy should be assessed in a study with a larger number of cases.

\section{REFERENCES}

1. Berman B, Bieley HC. Adjunct therapies to surgical management of keloids. Dermatol Surg 1996;22:126-30.

2. Stahl S, Barnea Y, Weiss J, et al. Treatment of earlobe keloids by extralesional excision combined with preoperative and postoperative "sandwich" radiotherapy. Plast Reconstr Surg 2010;125:135-41.

3. Botwood N, Lewanski C, Lowdell C. The risks of treating keloids with radiotherapy. Br J Radiol 1999;72:1222-4.

4. Wang Y, Qi Z, Wang X. Dermis reconstruction and dermis fat graft through an intraoral incision: a new method to correct the furrowed philtral column deformity in lesser-form cleft lip. Cleft Palate Craniofac J 2014;51:184-8.

5. Son D, Lee HG, Han KH, et al. Radiation therapy following total keloidectomy; a preliminary report. J Korean Soc Plast Reconstr Surg 2005;32:717-22.

6. Ogawa R, Miyashita T, Hyakusoku H, et al. Postoperative radiation protocol for keloids and hypertrophic scars: statistical analysis of 370 sites followed for over 18 months. Ann Plast Surg 2007;59:688-91.

7. Ogawa R, Mitsuhashi K, Hyakusoku H, et al. Postoperative electron-beam irradiation therapy for keloids and hypertrophic scars: retrospective study of 147 cases followed for more than 18 months. Plast Reconstr Surg 2003;111:547-53.
8. Niessen FB, Spauwen PH, Schalkwijk J, et al. On the nature of hypertrophic scars and keloids: a review. Plast Reconstr Surg 1999;104:1435-58.

9. Ogawa R, Huang C, Akaishi S, et al. Analysis of surgical treatments for earlobe keloids: analysis of 174 lesions in 145 patients. Plast Reconstr Surg 2013;132:818e-825e.

10. Ragoowansi R, Cornes PG, Moss AL, et al. Treatment of keloids by surgical excision and immediate postoperative single-fraction radiotherapy. Plast Reconstr Surg 2003;111: 1853-9.

11. Lo TC, Seckel BR, Salzman FA, et al. Single-dose electron beam irradiation in treatment and prevention of keloids and hypertrophic scars. Radiother Oncol 1990;19:267-72.

12. Dinh Q, Veness M, Richards S. Role of adjuvant radiotherapy in recurrent earlobe keloids. Australas J Dermatol 2004; 45:162-6.

13. Kovalic JJ, Perez CA. Radiation therapy following keloidectomy: a 20-year experience. Int J Radiat Oncol Biol Phys 1989; 17:77-80.

14. Levy DS, Salter MM, Roth RE. Postoperative irradiation in the prevention of keloids. AJR Am J Roentgenol 1976;127: 509-10.

15. Veen RE, Kal HB. Postoperative high-dose-rate brachytherapy in the prevention of keloids. Int J Radiat Oncol Biol Phys 2007;69:1205-8.

16. Kal HB, Veen RE, Jurgenliemk-Schulz IM. Dose-effect relationships for recurrence of keloid and pterygium after surgery and radiotherapy. Int J Radiat Oncol Biol Phys 2009; 74:245-51.

17. Hoffman S. Radiotherapy for keloids. Ann Plast Surg 1982; 9:265.

18. Ogawa R, Yoshitatsu S, Yoshida K, et al. Is radiation therapy for keloids acceptable? The risk of radiation-induced carcinogenesis. Plast Reconstr Surg 2009; 124:1196-201. 